Draft Version OCtOBER 31, 2018

Preprint typeset using $\mathrm{LATE}_{\mathrm{E}} \mathrm{X}$ style emulateapj v. 08/22/09

\title{
FORMATION OF THE FIRST NUCLEAR CLUSTERS AND MASSIVE BLACK HOLES AT HIGH REDSHIFT
}

\author{
B. DeVECCHI \\ Dipartimento di Fisica G. Occhialini, Università degli Studi di Milano Bicocca, Piazza della Scienza 3, 20126 Milano, Italy \\ AND \\ M. VOLONTERI \\ Astronomy Department, University of Michigan, 500 Church Street, Ann Arbor, MI, 48109, USA \\ Draft version October 31, 2018
}

\begin{abstract}
We present a model for the formation of massive black holes $\left(\sim 1000 \mathrm{M}_{\odot}\right)$ due to stellar-dynamical processes in the first stellar clusters formed at early cosmic times $(z \sim 10-20)$. These black holes are likely candidates as seeds for the supermassive black holes detected in quasars and nearby quiescent galaxies. The high redshift black hole seeds form as a result of multiple successive instabilities that occur in low metallicity $\left(Z \sim 10^{-5} Z_{\odot}\right)$ protogalaxies. We focus on relatively massive halos at high redshift $\left(T_{\text {vir }}>10^{4} \mathrm{~K}, z \gtrsim 10\right)$ after the very first stars in the Universe have completed their evolution. This set of assumptions ensures that (i) atomic hydrogen cooling can contribute to the gas cooling process, (ii) a UV field has been created by the first stars, and (iii) the gas inside the halo has been mildly polluted by the first metals. The second condition implies that at low density $\mathrm{H}_{2}$ is dissociated and does not contribute to cooling. The third condition sets a minimum threshold density for fragmentation, so that stars form efficiently only in the very inner core of the protogalaxy. Within this core, very compact stellar clusters form. The typical star cluster masses are of order $10^{5} \mathrm{M}_{\odot}$ and the typical half mass radii $\sim 1$ pc. A large fraction of these very dense clusters undergo core collapse before stars are able to complete stellar evolution. Runaway star-star collisions eventually lead to the formation of a very massive star, leaving behind a massive black hole remnant. Clusters unstable to runaway collisions are always the first, less massive ones that form. As the metallicity of the Universe increases, the critical density for fragmentation decreases and stars start to form in the entire protogalactic disk so that i) accretion of gas in the centre is no longer efficient and ii) the core collapse timescale increases. Typically a fraction $\sim 0.05$ of protogalaxies at $z \sim 10-20$ form black hole seeds, with masses $\sim 1000-2000 \mathrm{M}_{\odot}$, leading to a mass density in seeds of a few $\simeq 10^{2} \mathrm{M}_{\odot} / \mathrm{Mpc}^{-3}$. This density allows enough room for black hole growth by accretion during the quasar epoch.

Subject headings: black hole physics - instabilities - stellar dynamics - galaxies: nuclei - galaxies:formation
\end{abstract}

\section{INTRODUCTION}

Supermassive black holes (BHs) are routinely detected in the centre of galaxies, both in nearby quiescent galaxies, and as the engines that power quasars and active galactic nuclei. Observationally, we can trace quasars until very high redshifts. Luminous quasars are detected in the Sloan survey (e.g. Fan et al. 2001) at $z \simeq 6$, corresponding to a time when the Universe was not even one billion years old. This implies that the first BH seeds must have formed earlier on. The currently favored scenario for $\mathrm{BH}$ seed formation relies on the remnants of the very first generation of metal-free stars (Population III, PopIII). The first stars are believed to form at $z \sim 20$ in halos which represent rare high peaks of the primordial density field. Simulations of the fragmentation of zerometallicity protogalaxies suggest a very top-heavy initial stellar mass function, and in particular the production of very massive stars with mass $>100 \mathrm{M}_{\odot}$. PopIII stars in the mass range $140 \leq m_{\star} \leq 260 \mathrm{M}_{\odot}$ are predicted to make pair-instability supernovae. If zero metallicity very massive stars form above $260 \mathrm{M}_{\odot}$, they will rapidly collapse to BHs with little mass loss (Fryer et al. 2001), leaving behind $\mathrm{BHs}$ with masses $\simeq 10^{2} \mathrm{M}_{\odot}($ Madau \&

Electronic address: bernadetta.devecchi@mib.infn.it
Rees 2001).

Although this path to $\mathrm{BH}$ seed formation seems very natural, large uncertainties exist on the final mass of PopIII stars. Even recent simulations (Gao et al. 2006) have not clarified if PopIII stars are indeed very massive, and in particular if they are above the threshold $\left(\simeq 260 \mathrm{M}_{\odot}\right)$ for $\mathrm{BH}$ formation (but see Freese et al. 2008, Spolyar et al. 2008, Natarajan et al. 2008). Furthermore, metal abundances in extremely metal poor Galactic halo stars, which are commonly thought to trace the enrichment products of the first generation of stars, are incompatible with the yield patterns of zero metallicity very massive stars $\left(\gtrsim 100 \mathrm{M}_{\odot}\right.$, Tumlinson et al. 2004).

Alternative routes to $\mathrm{BH}$ seed formation have been explored (Haehnelt \& Rees 1993, Loeb \& Rasio 1994, Eisenstein \& Loeb 1995, Bromm \& Loeb 2003, Koushiappas et al. 2004, Begelman, Volonteri \& Rees 2006, Lodato \& Natarajan 2006), typically exploiting gas-dynamical processes in metal-free galaxies. Gravitational instabilities can indeed lead to a vigorous gas inflow into the very central region, supplying the necessary matter for the formation of $\mathrm{BH}$ seeds. The typical conditions that lead to efficient gas infall and $\mathrm{BH}$ seed formation can be summarized as (i) the host is massive enough that the virial temperature exceeds $T_{\text {vir }}>10^{4} \mathrm{~K}$, so that gas is 
able to cool and collapse via atomic hydrogen cooling. (ii) Molecular hydrogen does not form as the gas cools and condenses. (iii) The gas has primordial composition, so that metal line cooling is non-existent.

However, massive halos $\left(T_{\text {vir }}>10^{4} \mathrm{~K}\right.$, masses $\sim$ $\left.10^{7} \mathrm{M}_{\odot}\right)$ are likely built-up from mini-halos $\left(T_{\text {vir }}<10^{4}\right.$ $\mathrm{K})$ that had collapsed earlier on. Some of these halos might have experienced PopIII star formation, and should be enriched with at least some trace amount of metals. Fragmentation and formation of low mass stars starts as soon as gas is polluted by metals created in the first PopIII stars. Efficient gas collapse, leading to $\mathrm{BH}$ seed formation, is mutually exclusive with star formation, as competition for the gas supply limits the mass available.

However this first episode of efficient star formation can foster the formation of very compact nuclear star clusters (Clark et al. 2008, Schneider et al. 2006) where star collisions can lead to the formation of a supermassive star, possibly leaving a $\mathrm{BH}$ remnant with mass in the range $\sim 10^{2}-10^{4} M_{\odot}$ (Omukai et al. 2008).

The possibility that an "intermediate-mass" BH could in principle form as a result of dynamical interactions in dense stellar systems is a long standing idea (Begelman and Rees 1978; Freitag et al. 2006b,a, Ebisuzaki et al. 2001; Portegies Zwart and McMillan 2002; Miller \& Hamilton 2002, Gurkan et al. 2004). During their lifetime collisional stellar systems evolve as a result of dynamical interactions. In an equal mass system the central cluster core initially contracts as the system attempts to reach a state of thermal equilibrium: energy conservation leads to a decrease in the core radius as evaporation of the less bound stars proceeds. As a result the central density increases and the central relaxation time decreases. The core then decouples thermally from the outer region of the cluster. Core collapse then speeds up as it is driven by energy transfer from the central denser region (Spitzer 1987). This phenomenon is greatly enhanced in multi-mass systems like realistic star clusters. In this case the gravothermal collapse happens on a shorter timescale as dynamical friction causes the more massive stars of mass $m$, to segregate in the centre on a time-scale $t_{\mathrm{df}}=(\langle m\rangle / m) t_{r h}$ (where $t_{r h}$ is the half mass relaxation timescale and $\langle m\rangle$ is the mean stellar mass in the cluster). If mass segregation sets in before the more massive stars evolve out of the main sequence ( $\sim 3 \mathrm{Myr}$ ), then a sub-system decoupled from the rest of the cluster can form, where star-star collisions can take place in a runaway fashion that ultimately lead to the growth of a very massive star (VMS) (Portegies Zwart et al. 1999).

Yungelson et al. (2008) study the fate of solar composition VMSs in the mass range $60-1000 M_{\odot}$. They find that all VMSs are likely to shed most of their mass via winds well before experiencing a supernova explosion. Solar composition VMSs are therefore expected to end their lives as objects less massive than $\sim 150 M_{\odot}$, collapsing into BHs with mass $\lesssim 150 M_{\odot}$ or exploding as pair-instability supernovae. The growth of a VMS should be much more efficient at low metallicity. Low metallicity can modify the picture in different ways. First, at sub-solar (but still not primordial) metallicity, all stars with masses $\geqq 40 M_{\odot}$ are thought to collapse directly into a black hole (Heger et al. 2003) without exploding as supernovae. Second, the mass loss due to winds is much more reduced in metal-poor stars, which greatly helps in increasing the mass of the final remnant.

In this paper we investigate the formation of BHs, remnants of VMS formed via stellar collisions in the very first stellar clusters at early cosmic times. We derive the properties of the ensuing $\mathrm{BH}$ population, that can represent the "seeds" of the supermassive BHs that we observe in today's galaxies. In the following we summarize the main features of our model:

- we focus on halos with virial temperatures $T_{v i r} \gtrsim$ $10^{4} \mathrm{~K}$ at $z>5$ after the first episode of star formation, hence with a low, but non-zero, metallicity

- low metallicity gas can fragment and form lowmass stars only if the gas density is above a certain threshold, $n_{\text {crit }, Z}$

- halos possess angular momentum, acquired through interaction with their neighbors, hence gas collapse ultimately leads to the formation of a disk

- if the forming disk is Toomre-unstable, instabilities lead to mass infall instead of fragmentation into bound clumps and global star formation in the entire disk

- the gas inflow increases the central density, and within a certain, compact, region $n>n_{\text {crit }, Z}$

- star formation ensues and a dense star cluster is formed

- if the star cluster goes into core collapse in $\lesssim 3 \mathrm{Myr}$ runaway collisions of star form a supermassive star, leading to a massive $\mathrm{BH}$ remnant.

We describe the physical mechanism for cluster formation in section 2, where we discuss the conditions leading to very compact stellar clusters in low metallicity $\left(Z \sim 10^{-5} Z_{\odot}\right)$ protogalaxies. We discuss dynamical instabilities (Section 2.1) driving gas towards the centre, and fragmentation occurring at high densities (Sections 2.2 and 2.3). The properties of the star clusters, VMSs and BHs are described in Sections 2.4 and 2.5. We present our results in Section 3 and discuss the implications of our model in Section 4.

\section{BH SEED FORMATION: MODEL SET-UP}

We start by considering a halo of mass $M_{h}$, with virial circular velocity $V_{h}$, virial radius $R_{h}$, angular momentum $J$. The rotational support can be quantified in terms of the spin parameter $\lambda \equiv J|E|^{1 / 2} / G M_{h}^{5 / 2}$, where $E$ is the total energy of the halo. We further assume that a fraction $m_{d} \sim 0.05$ of the halo mass is in a gaseous baryonic component than can cool and condense. The collapsing mass will then be $m_{d} M_{h}$. The angular momentum of the collapsing baryons will be a fraction $j_{d}$ of the halo angular momentum, with $j_{d} / m_{d}=1$ if the specific angular momentum of baryons is conserved during collapse. In this work we focus on relatively massive halos $\left(T_{\text {vir }}>10^{4} \mathrm{~K}\right.$, ensuring that gas can cool by atomic 
hydrogen) after the birth of the first stars in the Universe. $10^{4} \mathrm{~K}$ halos are likely build up by smaller halos that have already experienced a first episode of star formation. We assume that the first PopIII stars have been able to affect the gas both radiatively (thus precluding subsequent $\mathrm{H}_{2}$ cooling in the halo) and chemically. The first condition implies that, at least at the low density at which the collapse starts, $H_{2}$ is dissociated and does not contribute to the cooling of the gas (see also Figures 9 and 10 in O'Shea \& Norman 2008). The second condition ensures that metals (and dust) in small quantities can cool down the gas efficiently only as the gas density reaches a critical threshold (see Section 2.2, and Figures 2, 4 and 5 in Smith et al. 2008). Jointly, the two conditions ensure that at first cooling is driven by atomic hydrogen only.

\subsection{Disk formation and mass inflow}

We describe the early evolution of the cooling and collapsing baryons in a simple way, assuming adiabatic response of the halo to gas cooling and disk formation (e.g., Mo, Mao \& White 1998, Oh \& Haiman 2002, Lodato \& Natarajan 2006). As we are assuming negligible $H_{2}$, the tenuous gas cools down by atomic hydrogen only until it reaches $T_{\text {gas }} \sim 4000 \mathrm{~K}$. At this point the cooling function of the atomic hydrogen drops by a few orders of magnitude, and contraction proceeds nearly adiabatically.

Given the presence of angular momentum, the contraction in the equatorial plane stops as the gas becomes rotationally supported, and the collapse ultimately leads to the formation of a disk. As pointed out by Mo, Mao \& White (1998), the properties of the disk can be determined by assuming a profile for the surface density $\Sigma$. As the mass assembles in the protodisk the surface density increases. If for the final configuration the Toomre parameter:

$$
Q=\frac{c_{s} \kappa}{\pi G \Sigma}<Q_{c}
$$

where $c_{s}$ is the sound speed, $\kappa$ is the epicyclic frequency and $Q_{c}$ is a critical value, then the disk becomes unstable, and it develops bar-like structures. We argue that if the destabilisation of the system is not too violent, instabilities lead to mass infall instead of fragmentation into bound clumps and global star formation in the entire disk (Shloshman et al. 1990, Lodato \& Natarajan 2006). This is the case if the inflow rate is below a critical threshold $\dot{M}_{\max }=2 \alpha_{c} \frac{c_{s}^{3}}{G}$ the disk is able to sustain (where $\alpha_{c} \sim 0.12$ describes the viscosity) and molecular and metal cooling are not important. We substantiate further our argument in Section 2.2. In the following, we discuss what happens to the disk if fragmentation is (at least initially) suppressed.

The classical analysis yields $Q_{c} \simeq 1$ for axi-symmetric disturbances. However a disk can develop large scale non-axisymmetric instabilities even for $Q>1$ so that a limit value $Q_{c} \sim 2$ is probably a better guess (Lodato 2008). We will assume in the following $Q_{c}=2$. The frequency of disk instabilities as a function of $Q_{c}$ is discussed in Lodato \& Natarajan (2007) and in Volonteri, Lodato \& Natarajan (2008), who showed that any $Q_{c}<1.5$ underestimates the $\mathrm{BH}$ population, even assuming a $100 \%$ of $\mathrm{BH}$ formation in unstable disks.
The torques induced by self-gravity can cause a relatively fast redistribution of gas within the disk: the gas shocks and loses angular momentum thus sinking to the centre of the system. As gas flows into the central region, the surface density in the outer part of the disk decreases until the Toomre parameter nears the critical value once again. At this point the infall stops. In this sense the instabilities described here are self-regulating and end up in a condition of marginal stability. Ultimately, the mass routed in the central part corresponds to the amount necessary for the outer disk to be marginally stable. Let us define this central mass condensation as a fraction $m_{a}$ of the halo mass, that corresponds to a baryonic mass $m_{a} M_{h}$.

A marginally stable, isothermal disk has the surface density profile consistent with a Mestel disk, with $\Sigma=$ $\Sigma_{0}\left(\frac{R}{R_{0}}\right)^{-1}$. This is our initial condition for the outer region of the disk. As the mass $m_{a} M_{h}$ is added to the mass already in place in the centre, the inner surface density profile steepens, $\Sigma \propto\left(\frac{R}{R_{0}}\right)^{-\gamma}$, with $\gamma>1$. Mineshige \& Umemura (1997) studied the collapse of an isothermal, rotationally supported, self-gravitating collapsing disk. The collapsing disk develops a different structure in the inner and outer part, and the collapse proceeds with time as follows:

$$
\Sigma(R) \sim \begin{cases}\Sigma_{i n}\left(\frac{R}{R_{0}}\right)^{-5 / 3}\left(\frac{t}{t_{0}}\right)^{2 / 3} & R<R_{t r} \\ \Sigma_{0}\left(\frac{R}{R_{0}}\right)^{-1} & R>R_{t r}\end{cases}
$$

where the transition radius $R_{t r}$ between the two profiles evolves linearly with time. As the transport associated with the development of gravitational instabilities can be well described in terms of an effective viscosity (Lodato \& Natarajan 2006), we take the result of Mineshige \& Umemura as a reference for our system and we assume that the inner profile steepens with $\gamma=5 / 3$ unless otherwise noted.

The final configuration of the structure would then be characterized by an outer surface density profile of a Mestel disk and a central denser region:

$$
\Sigma(R) \sim \begin{cases}\Sigma_{i n}\left(\frac{R}{R_{0}}\right)^{-\gamma} & R<R_{t r} \\ \Sigma_{0}\left(\frac{R}{R_{0}}\right)^{-1} & R>R_{t r}\end{cases}
$$

where $\Sigma_{i n}$ can be written as $\Sigma_{0}\left(R_{0} / R_{t r}\right)^{1-\gamma}$ by imposing continuity at $R_{t r}$. At this point the parameters of the final disk are $\Sigma_{0}, R_{0}, R_{t r}$ and the fractional mass of the halo $m_{a}$ that participate to the infall. They can be determined by imposing the conditions of mass and angular momentum conservation, $Q=Q_{c}$ (for $\left.R>R_{t r}\right)$ and by imposing that a mass $m_{a} M_{h}$ is added to that already in place inside $R_{t r}$. For simplicity we assume that the dark matter halo hosting the protogalaxy follows an isothermal profile. The resulting disk properties are as follows:

$$
\Sigma_{0}=\frac{10 m_{d}\left(m_{d} / j_{d}\right)^{2} H_{z} V_{h}\left(1-m_{a} / m_{d}\right)^{3}}{16 \pi G \lambda^{2}}
$$




$$
\begin{gathered}
R_{0}=\frac{2 \sqrt{2}\left(j_{d} / m_{d}\right) \lambda R_{h}}{\left(1-m_{a} / m_{d}\right)} \\
m_{a}=m_{d}\left[1-\sqrt{\frac{8 \lambda}{m_{d} Q_{c}} \frac{j_{d}}{m_{d}}\left(\frac{T_{\text {gas }}}{T_{\text {vir }}}\right)^{1 / 2}}\right] \\
R_{t r}=\frac{m_{a} M_{h}}{4 \pi \Sigma_{0} R_{0}}
\end{gathered}
$$

where $H_{z}$ is the Hubble constant at redshift $z$. The vertical structure of the disk is determined by solving the equation for hydrostatic equilibrium. For a disk that is isothermal and self-gravitating, this implies a z-dependency for the density $\propto \cosh ^{-2}(h / H)$ where $H$ is the vertical scale-height and it is equal to $H(R)=$ $c_{s}^{2} / \pi G \Sigma(R)$. We can therefore express the inner disk density as:

$$
n(R, h)=n_{0}\left(\frac{R}{R_{0}}\right)^{-5 / 3} \cosh ^{-2}(h / H(R)),
$$

where $n_{0}$ is a function of the disk parameters, $\Sigma_{0}, R_{0}$ and $\gamma$ (see Appendix A).

\subsection{Fragmentation of the disk}

A necessary condition for the inflow process described in the previous Section is that star formation does not take place in the entire disk. If this happens, the gas that would otherwise flow into the central region, is consumed as it is converted into stars.

Rice et al. (2005) proposed that fragmentation in a thin disk sets in when the gravitationally induced stress exceeds a critical value. Describing angular momentum transport in terms of the $\alpha$ prescription for viscous dissipation (i.e. that the torque strength can be expressed in terms of $\alpha$, Shakura \& Sunyaev 1973), then the critical threshold for fragmentation $\alpha_{c}$ determines how much angular momentum can be transported in a steady state. In this sense the fragmentation boundary is ultimately due to the inability of the disk to redistribute angular momentum on a sufficiently short timescale. In the classical analysis (Gammie 1996) fragmentation develops as the condition $t_{c o o l}=t_{d y n}$ is reached. In the framework of Rice et al. (2005) we can expect that the same effect develops if the mass inflow from the halo induces too strong a stress. Lodato \& Natarajan (2006) apply this framework to disks of primordial composition. By requiring the mass-accretion rate from the halo $\dot{M}_{h}=m_{d} \frac{V_{h}^{3}}{G}$ to be less than the maximum value $\dot{M}_{\max }=2 \alpha_{c} \frac{c_{s}^{3}}{G}$ the disk is able to sustain, they argue that to avoid fragmentation it must be

$$
\frac{T_{v i r}}{T_{\text {gas }}}<\left[\frac{4 \alpha_{c}}{m_{d}} \frac{1}{1+m_{a} / m_{d}}\right]^{2 / 3} .
$$

where $\alpha_{c} \sim 0.12$ (Clarke et al. 2007) is the critical value for fragmentation.
We assume $T_{\text {gas }}=4000 \mathrm{~K}$, which corresponds to $\dot{M}_{\max } \sim 10^{-2} \mathrm{M}_{\odot} \mathrm{yr}^{-1}$. The joint conditions, $m_{a}>0$ (Equation 6) and Equation 9 then impose an upper limit to the virial temperature $T_{v i r} \leqq 1.8 \times 10^{4} \mathrm{~K}$. We therefore consider halos with $10^{4} \mathrm{~K} \leqq T_{v i r} \leqq 1.8 \times 10^{4} \mathrm{~K}$.

By considering these gas and virial temperatures we can ensure that the fragmentation threshold is not attained at least at $R>R_{t r}$. However, $T_{\text {gas }}=4000 \mathrm{~K}$ requires the absence of coolants other than atomic hydrogen. If $H_{2}$ contributes efficiently as a coolant, the gas temperature drops, and Equation 9 implies that massive halos are subject to strong fragmentation. For instance, if gas cools to $T_{\text {gas }} \sim 200 \mathrm{~K}$, only halos with $T_{v i r} \leqq 1000$ $\mathrm{K}$ satisfy Equation 9, At redshift $\sim 20$ this corresponds to halo masses around a few $10^{5} \mathrm{M}_{\odot}$.

The issue of $\mathrm{H}_{2}$ cooling suppression in the presence of a strong UV field has been addressed in a number of recent studies on the first galaxies. Even if a strong UV background is not already in place, a "local" UV field can be established by the presence of PopIIIs in surrounding halos. Eventually, $\mathrm{H}_{2}$ can be suppressed by previous PopIII star formation in the smaller systems assembled to form the final $T_{v i r} \sim 10^{4} \mathrm{~K}$ halo (see Ciardi (2008) or Ciardi \& Ferrara (2005) for a discussion on this issue). O'Shea \& Norman (2008) have shown that halos with $T_{v i r} \sim 10^{4}$ $\mathrm{K}$ embedded in a UV background with strength higher than $\sim 3 \times 10^{-23} \mathrm{erg} \mathrm{s}^{-1} \mathrm{~cm}^{-2} \mathrm{~Hz}^{-1} \mathrm{sr}^{-1}$ are not subject to efficient cooling and fragmentation in the disk (see their Fig. 9b) and it is only in the central region $(\mathrm{r}<10$ pc) that $T_{\text {gas }}$ drops down to $\sim 10^{3} \mathrm{~K}$, leading to the formation of a central single massive star born in isolation. Halos subject to a weaker UV field show the same radial behavior but shifted at lower temperature. If $H_{2}$ cooling in fact does not act on large scales, it does not affect the inflow process described in Section 2.1 so that material can still be transported to small distances by dynamical instabilities.

In metal-free conditions, the gaseous density in the central region increases until $\mathrm{H}_{2}$ is activated (O'Shea \& Norman 2008, Omukai et al. 2008, and references therein) and the formation of a Pop III star can proceed. On the other hand, if the gas has been enriched to a certain level, fragmentation can take place and an entire stellar cluster is formed (Clark et al. 2008). It is this last situation that we want to examine in more details, i.e. the possibility that a cluster of stars is formed instead of a single massive star.

\subsection{Critical metallicity for fragmentation}

Various authors have suggested that the presence of a certain amount of metals is the key ingredient in order to produce efficient fragmentation (Schneider et al. 2006; Bromm et al. 1999, 2002; Omukai et al. 2008; Clark et al. 2008 and references therein). Let us define a critical metallicity $Z_{\text {crit }}$ where transition from PopIII to "normal" star formation occurs. $Z_{\text {crit }}$ depends on the coolants at work. Bromm et al. (2001) simulated the collapse of a halo of $2 \times 10^{6} M_{\odot}$ at different values of $Z$. They estimate $Z_{\text {crit }} \sim 10^{-3.5}$ and show that when $Z>Z_{\text {crit }}$ a rotationally supported disk that fragments vigorously can form. More recently, Clark et al. (2008) simulate the collapse of a rotating cloud polluted by dust. They show that a tightly packed cluster of protostars is 
formed in the centre.

To estimate the value of $Z_{\text {crit }}$ at which gas start to fragment one can compare the cooling rate $\Lambda_{\text {cool }}$ to the adiabatic heating rate $\Gamma_{a d}$. Fragmentation requires the $\Lambda_{\text {cool }} \geqq \Gamma_{a d}$. Once this happens, the temperature starts to decrease as the density increases until cooling stops to be efficient. The condition $\Gamma_{a d}<\Lambda_{\text {cool }}$ in fact constraints the metallicity at a given temperature and density to be greater than a critical value $Z_{\text {crit }}$. For an initially isothermal gas this condition provides a relationship between metallicity and density. Equivalently, if the gas is characterized by a fixed metallicity $\mathrm{Z}$, then only those regions with density greater than a given threshold $n_{\text {crit }, Z}$ are able to cool down efficiently and fragment into stars. This is the condition that we apply to collapsing disks to determine if they can develop into stellar clusters.

Santoro et al. (2006) studied the condition for fragmentation in a low metallicity Universe assuming that PopIII stars are the main sources of pollution. The $Z_{\text {crit }}-n_{\text {crit }, Z}$ relation depends on the ratio between the different species of coolants (see Figure 9 in Santoro et al. 2006). In all cases, the critical metallicity increases with decreasing density untill $n$ reaches the critical value for collisional de-excitation of the dominant coolants. We adopt as a reference the curve corresponding to PopIII stars in the intermediate mass range (mass range 185$205 \mathrm{M}_{\odot}$; however the solar abundance ratio produces a similar pattern as well, cfr. solid and dotted curves in Figure 10 in Santoro \& Shull 2006). We discuss how our results depend on the specific $Z_{c r i t}-n_{\text {crit }, Z}$ relation in section 3.2.2.

The metallicity of gas in a given halo most probably depends on its mass and on the redshift. The cosmic metallicity history (MEH) has been investigated both from an observational and a theoretical prospective (Scannapieco et al. 2003, Tornatore et al. 2006, Savaglio 2006, Savaglio et al. 2005, Prochaska et al. 2003, Prochaska et al. 2007, Kulkarni et al. 2005, Li 2007). The observed mean metallicity decreases with redshift, but the rate of decrease depends on the type of sources studied. From studies of QSOs-DLAs, $Z / Z_{\odot}$ scales as $10^{-\beta z}$ with $\beta=0.36$ (Li 2007), while measurement based on GRBs point towards a shallower decline, so that at $z \sim 3-4$ already $Z=10^{-1} Z_{\odot}$ (Savaglio 2006). The differences in the slope and in the normalization are often ascribed to different histories of metal enrichment in QSOs and GRBs hosts (Li 2007 and references therein).

We model the MEH based on fits presented in Li 2007, that we refer to for additional details. In our reference model we adopt $\beta=0.36$, that is $Z / Z_{\odot}=0.35 \times 10^{-0.36 z}$. The case $\beta=0.18$, leading to $Z / Z_{\odot}=0.63 \times 10^{-0.18 z}$, is discussed in section 3.2.3. We further allow a logarithmically uniform scatter in $Z$ of $\Delta \log (Z)=1.5$, based on the observed scatter in $Z / Z_{\odot}$ from measurements of the QSO-DLAs. We wish to stress that our treatment of MEH is highly simplified. In a forthcoming paper we will determine self-consistently the evolution of the $\mathrm{MEH}$ from stellar winds pollution.

\subsection{Stellar cluster formation}

The central star cluster forms within the region where gas at a given metallicity has reached the critical density for fragmentation. Given a specific density profile (Equation 2) the requirement $n>n_{\text {crit }, Z}$ translates into a condition $R<R_{S F}$, where we define $R_{S F}$ as the radius where the density in the plane $h=0$, reaches the value $n_{\text {crit }, Z}$ for the gas metallicity $Z$, that is:

$$
n\left(R=R_{S F}, h=0\right)=n_{\text {crit }, Z} .
$$

As long as $n\left(R_{t r}, 0\right)<n_{\text {crit }, Z}, n(R, h)$ is defined by Equation 8 and the radius within which gas fragments and stars form can be written as:

$$
R_{S F}=R_{t r}\left[\frac{\Sigma_{0}}{c_{s}} \frac{R_{0}}{R_{t r}} \sqrt{\frac{\pi G}{2 \mu m_{H} n_{c r i t}, Z}}\right]^{1 / \gamma}
$$

where the metallicity dependence is included implicitly in $n_{c r i t, Z}$ (see Appendix A), $\mu$ is the molecular weight and $m_{H}$ is the proton mass. The half mass radius can be expressed as a function of the cluster radius in a very simple way, as

$$
R_{h}=2^{1 /(\gamma-1)} R_{S F} .
$$

For a given $\gamma$ the mass in stars of the cluster can be calculated as

$$
\begin{aligned}
M_{c l} & =\epsilon_{S F} 2 \pi \int_{0}^{R_{S F}} \Sigma(R) R d R \\
& =\epsilon_{S F}\left[\frac{2 \pi}{2-\gamma} \frac{\Sigma_{0}^{2} R_{0}^{2}}{c_{s}} \sqrt{\frac{\pi G}{2 \mu m_{u} n_{c r i t}}}\right]^{\frac{2-\gamma}{\gamma}}\left(\frac{m_{a} M_{h}}{\gamma-1}\right)^{\frac{2 \gamma-2}{\gamma}}
\end{aligned}
$$

where $\epsilon_{S F}$ is the fraction of gas converted into stars. We assume $\epsilon_{S F}=0.25$, consistently with the star formation efficiency in the low redshift Universe (Lada \& Lada 2003). Given the metallicity, $Z$, it is now possible to determine the extent of the region inside which star formation is allowed. The cluster properties for a given halo, are therefore uniquely described for any given metallicity.

At high metallicities, the case $n\left(R_{t r}, 0\right)>n_{\text {crit }, Z}$ becomes common. This implies $R_{S F}>R_{t r}$, that is, star formation takes place in the region of the disk where no inflow is taking place. In other words, once the gas reaches $R_{S F}$, it is no longer able to be routed efficiently in the inner region as star formation starts to consume gas. In the remainder of the paper we will assume conservatively that if $R_{S F}>R_{t r}$ no cluster formation occurs, as stars are formed in the disk rather than in the central compact region.

\subsection{Runaway instability of the central cluster}

The conditions under which "mass segregation instability" can occur have been investigated in a series of papers, focusing on clusters in the present-day Universe. A successful core collapse requires that the core collapse time be less than the main sequence lifetime of the most massive stars (mass losses from supernovae expand the core and increase interaction times). The main sequence lifetime of massive stars asymptotes to about 2.5 Myr because all stars go off the main sequence when they have consumed about $15 \%$ of their hydrogen, and for high-mass stars with luminosities approaching Eddington, $L \propto M$ (not $M^{3.5}$ as is the case for lower masses) and then the lifetime $\propto 0.15 M / L \sim$ const.

With a Monte Carlo code, Gürkan et al. (2004) found that typically the mass of the collapsing core is $10^{-3}$ times that of the entire cluster. Similarly, Portegies 
Zwart and McMillan (2002) related the mass of the VMS with the parameters of the cluster taking into account both numerical simulations and analytical arguments. Portegies Zwart \& McMillan (2002) have shown that core collapse occurs on a timescale

$$
\begin{aligned}
t_{c c} \simeq & 3 \operatorname{Myr}\left(\frac{R_{\mathrm{h}}}{1 \mathrm{pc}}\right)^{3 / 2}\left(\frac{M_{c l}}{5 \times 10^{5} M_{\odot}}\right)^{1 / 2} \times \\
& \left(\frac{10 M_{\odot}}{\langle m\rangle}\right)\left(\frac{8.5}{\ln \lambda_{C}}\right),
\end{aligned}
$$

where $\ln \lambda_{C}$ is the Coulomb logarithm of dynamical friction (Binney \& Tremaine 1987). The same simulations also find that the mass of the VMS can reach values as high as $10^{3} M_{\odot}$ (see also Freitag et al. 2006b). The final mass of the VMS however depends on complex phenomena related to both the dynamics and hydrodynamics of the collisions. At solar metallicity the growth of a star with mass greater than $\sim 100 M_{\odot}$ can be highly problematic as mass loss occurs both during the main sequence phase and at the end of the evolution when the star collapses into a BH. The growth of a VMS should in principle be much more efficient for metal-poor stars as in this case as mass loss should be strongly reduced. Recent models of stellar evolution at $Z \sim 10^{-5} Z_{\odot}$ have shown that mass loss due to stellar winds during the main sequence phase is almost unimportant and that the main contribution to the reduction of the stellar mass is due to the effect of rotation. This can reduce the mass of the star by a factor of order 2-4 (Meynet et al. 2008). If the final mass achieved by the VMS is greater than $\sim 260 \mathrm{M}_{\odot}$, then after the main sequence it collapses into a $\mathrm{BH}$ retaining most of its mass (Heger et al. 2003).

To estimate of the final mass of the VMS we follow the treatment outlined in Portegies Zwart (2002). We assume that the mass of the $\mathrm{BH}$ seed, $M_{B H}$, corresponds to the final mass of the VMS:

$$
M_{B H}=m_{*}+4 \times 10^{-3} M_{c l} f_{c} \ln \lambda_{C} \ln \left(\frac{t_{M S}}{t_{c c}}\right)
$$

Here $t_{M S}=3 \mathrm{Myr}$ is the main sequence lifetimes of massive ( $>40 M_{\odot}$, Hirschi 2007) stars, $m_{*}$ is the initial mass of the seed star that experiences runaway growth and $f_{c}$ is a factor used to calibrate the analytical expectation with direct numerical simulations. Portegies Zwart \& McMillan (2002) find $f_{c}=0.2$ for $\ln \left(\lambda_{C}\right)=\ln \left(0.1 M_{c l} /\langle m\rangle\right)$. We adopt the same values as Portegies Zwart \& McMillan (2002) for both parameters, further assuming $m_{*}=\langle m\rangle=10 \mathrm{M}_{\odot}$ consistent with the characteristic stellar mass in $10^{7-8} \mathrm{M}_{\odot}$ halos at redshift $\sim 10$ (see Figures 2 and 3 in Clarke \& Bromm 2003).

It is important to stress that Equation 15 provides an upper limit to $M_{B H}$. However, once the seed is born, the $\mathrm{BH}$ is still embedded into a dense cluster of stars. Even if its growth has been limited before the collapse of the VMS, the remnant can still gain mass by accretion of stars (for the growth rate of a massive $\mathrm{BH}$ hosted in a cluster of stars see for example the models of Marchant \& Shapiro 1979). If the combination of star formation and stellar feedback of the cluster stars do not deplete of gas the inner region, this surviving gas can supply an additional reservoir of material for $\mathrm{BH}$ growth. Both this
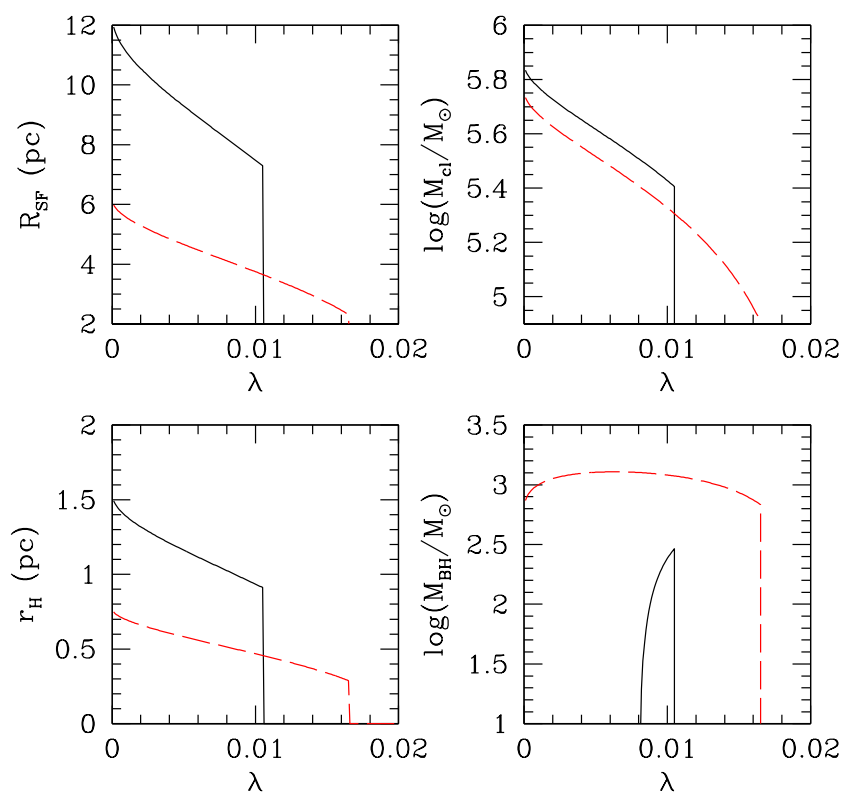

FIG. 1.- Cluster properties as a function of the spin parameter $\lambda \equiv J|E|^{1 / 2} / G M_{h}^{5 / 2}$ (i.e., the fraction of halo support given by rotation) for 2 critical densities for fragmentation, $n_{\text {crit }}=10^{3}$ $\mathrm{cm}^{-3}$ (solid line), and $n_{\text {crit }}=10^{4} \mathrm{~cm}^{-3}$ (dashed line). Curves are truncated when $R_{S F}$ equals $R_{t r}$.
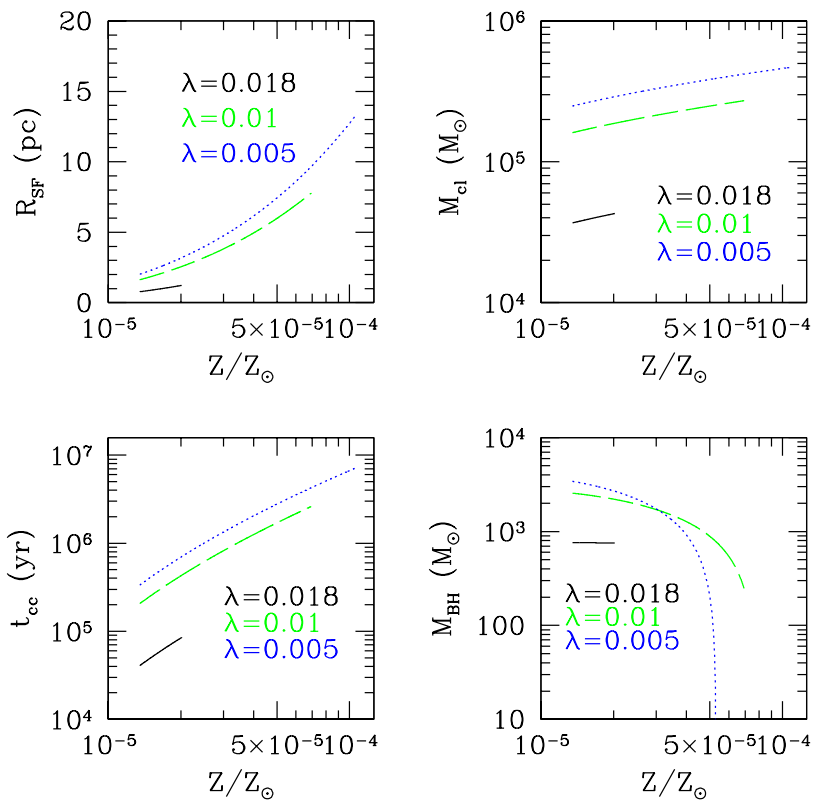

FIG. 2.- Cluster properties as a function of metallicity for 3 spin parameters: $\lambda=0.018$ (solid line), $\lambda=0.01$ (dashed line), $\lambda=$ 0.015 (dotted line). Each curve begins at low metallicity when halos starts star formation and ends when $R_{S F}$ equals $R_{t r}$; this happens at different critical metallicities for different spin parameters. Here the $Z_{\text {crit }}-n_{\text {crit }, Z}$ relationship follows the intermediate PopIII mass case in Santoro et al. 2006

processes can contribute to bring the $\mathrm{BH}$ mass to values as high as Equation [15] would suggest.

Clusters forming in unstable disks might have some degree of rotation. In this case the gravogyro instability (Inagaki \& Hachisu 1978) could contribute to accelerate 
the core collapse. The gravogyro instability is believed to occur in systems that exhibit a radial gradient of the angular speed. In analogy with viscous transport, angular momentum is transfered outwards. The core of the star cluster then contracts because of a deficit in the centrifugal force. Ernst et al. (2007) show that the gravogyro instability occurs in clusters with equal-mass stars, but in systems with two-mass components the effect of rotation seems negligible, as mass segregation and rotation compete in leading the evolution of the stellar cluster. For this reason we neglect its contribution in this work.

\subsection{Cluster Properties}

Various cluster properties: size (Equation 11), halfmass radius (Equation 12), mass (Equation 13) are shown in Figure [1] as a function of the spin parameter for two representative values of the critical density for fragmentation. We also present $\mathrm{BH}$ masses (Equation $15)$, where we have further imposed the condition $t_{c c}<3$ Myr.

As $\lambda$ increases, less mass inflows within $R_{t r}$, and $R_{S F}$ is reached at smaller radii at a given $n_{\text {crit }}$. Therefore $R_{S F}$ and $M_{c l}$ decrease with increasing $\lambda$, contrary to the disk size $R_{0}$. Note how at low critical densities (corresponding to high metallicities) only a very small fraction of systems can undergo core collapse, as on the one hand cluster formation is suppressed for large $\lambda$ (as $R_{S F}>R_{t r}$ ), on the other hand clusters are too massive and large at small $\lambda$ for fulfilling the condition $t_{c c}<3 \mathrm{Myr}$. Clusters undergoing core collapse have preferentially large spin parameters, within the region where cluster form. Figure 2 shows cluster sizes, masses, core collapse timescales (Equation 13) and BH masses as a function of metallicity. As the metallicity increases, the critical density for fragmentation decreases and cluster formation is eventually suppressed when $n\left(R_{t r}, 0\right)>n_{\text {crit }, Z}\left(R_{S F}>R_{t r}\right)$. At metallicities below $10^{-5} Z_{\odot}$ fragmentation is impossible for the specific choice of the $Z_{c r i t}-n_{\text {crit }, Z}$ relationship (intermediate PopIII mass case in Santoro et al. 2006).

The parameter space (virial temperature, spin parameter) where the multiple instabilities are efficient is shown in Figure 3. Here we select halos with $T_{\text {vir }}>10^{4} \mathrm{~K}$ at $z=12$, and derive the disk and cluster properties, assuming a single critical density for fragmentation (from $10^{3} \mathrm{~cm}^{-3}$ to $10^{4} \mathrm{~cm}^{-3}$ ). The higher the critical density for fragmentation (i.e., the lower the metallicity, see Section 2.3) the more compact are the clusters, and the shorter is $t_{c c}$. When $n_{c r i t, Z}<10^{3} \mathrm{~cm}^{-3}$ no clusters can undergo core collapse in less than $3 \mathrm{Myr}$. When $n_{\text {crit }, Z}>10^{4}$ $\mathrm{cm}^{-3}$ all forming clusters undergo core collapse in less than 3 Myr.

\section{RESULTS}

\subsection{Summary of the general procedure}

We first summarize the procedure taken in order to determine the properties of the $\mathrm{BH}$ seed population. We calculate the mass of halos that at redshift $z$ correspond to virial temperatures $10^{4} \mathrm{~K} \leqq T_{\text {vir }} \leqq 1.8 \times 10^{4}$ K (Barkana \& Loeb 2001) and we determine their frequency using a modified version of the Press \& Schechter formalism (Sheth \& Tormen 1999) in a WMAP5 cosmology (Dunkley et al. 2008). To each halo we assign a value of the spin parameter, $\lambda$, extracted from the probability
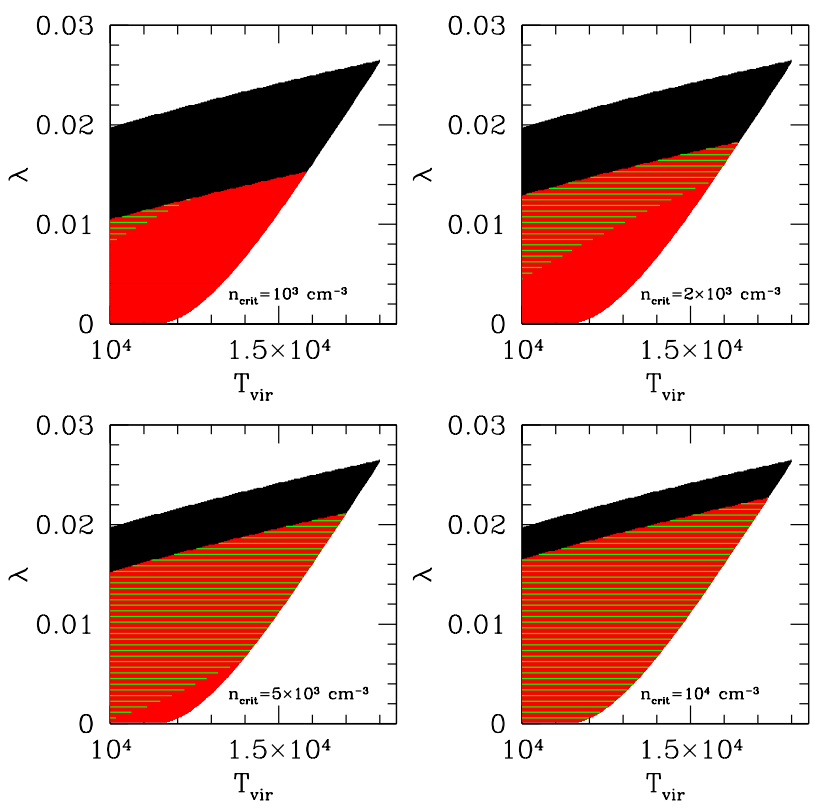

FIG. 3.- Parameter space (virial temperature, spin parameter) for cluster and $\mathrm{BH}$ formation. Here we select halos with $T_{v i r}>10^{4}$ $\mathrm{K}$ at $z=12$, and derive the disk and cluster properties, assuming a single critical density for fragmentation (top-left: $10^{4} \mathrm{~cm}^{-3}$, topright: $2 \times 10^{4} \mathrm{~cm}^{-3}$, bottom-left: $2.5 \times 10^{4} \mathrm{~cm}^{-3}$, bottom-right: $3 \times 10^{4} \mathrm{~cm}^{-3}$; for the chosen $Z_{c r i t}-n_{\text {crit }, Z}$ relationship these densities correspond respectively to $\left.\log \left(Z / Z_{\odot}\right)=-4.2 ;-4.3 ;-4.4 ;-4.5\right)$. The black shaded area shows the range of temperatures and spin parameters where disks are Toomre unstable and the joint conditions, $m_{a}>0$ (Equation [6) and Equation 9] are fulfilled. The lighter shaded area selects the systems where $R_{S F}<R_{t r}$. The hatched area picks the subsample of clusters where $t_{c c}<3 \mathrm{Myr}$, where VMSs and $\mathrm{BH}$ seeds can form.

distribution found in the Millennium simulations (Bett et al. 2007):

$$
P(\log \lambda)=A\left(\frac{\lambda}{\lambda_{0}}\right)^{3} \exp \left[-\zeta\left(\frac{\lambda}{\lambda_{0}}\right)^{3 / \zeta}\right]
$$

where $\lambda_{0}=0.0043$ is the peak location, $\zeta=2.509$ and the normalization reads $A=3 \ln 10 \zeta^{\zeta-1} / \Gamma(\zeta)$, with $\Gamma$ being the gamma function. This set of assumptions allows us to calculate the initial disk properties, $\Sigma_{0}, R_{0}, Q_{c}, R_{t r}$ and $m_{a}$.

We assign to each halo a metallicity, $Z$ by extrapolating at higher redshift the fit to the observational constraints of the $\mathrm{MEH}\left(Z \propto 10^{-\beta z}\right)$, taking also into account the observed metallicity scatter. We then calculate $n_{\text {crit }, Z}$ from a given $Z_{\text {crit }}-n_{\text {crit }, Z}$ relation. If a protogalaxy has $Q<Q_{c}$ we determine the properties of the stellar cluster $\left(R_{S F}, M_{c l}\right.$ and $\left.R_{h}\right)$. We then check if the cluster can develop runaway instability via Equation 14. and we select the systems where $t_{c c}<3 \mathrm{Myr}$. For these unstable clusters we calculate the expected mass of the seed BH from Equation 15. In Table 1 we summarize all the different cases we describe in the next sections.

We now discuss the properties that nuclear clusters possess at birth and the resulting $\mathrm{BH}$ seed population. We start describing our reference model, A, in Section 3.1 while in Section 3.2 we discuss how our results depend on PopIII stars metallicity patterns and on the rate of 


\begin{tabular}{|c|c|c|c|}
\hline Run & MEH & $Z_{\text {crit }}-n_{\text {crit }, Z}$ & $\Delta \log (Z)$ \\
\hline A & 0.36 & 2 & 1.5 \\
B & 0.18 & 2 & 1.5 \\
C & 0.36 & 2 & 0 \\
D & 0.36 & 1 & 1.5 \\
E & 0.36 & 3 & 1.5 \\
\hline
\end{tabular}

TABLE 1

List OF THE SIMULATIONS: LABEL OF THE RUN, POWER-LAW INDEX OF THE METALLICITY DEPENDENCE BY REDSHIFT, POPIII YIELD CURVES (1, 2 AND 3 REFER TO LOWER, CENTRAL AND UPPER CURVES OF Figure 10 in SANTORO \& Shull (2006) RESPECTIVELY), ALLOWED VARIATION (LOGARITHMICALLY UNIFORMLY DISTRIBUTED) IN $Z$.

metal enrichment of the Universe.

\subsection{Model A}

The first halos reach the critical metallicity for fragmentation at redshift $\sim 14$. This is also when the first stellar clusters form. The first clusters have masses of the order of $10^{5} \mathrm{M}_{\odot}$ and $R_{h} \sim 0.5-1 \mathrm{pc}$; in such compact clusters, core collapse starts early $\left(\left\langle t_{c c}\right\rangle \sim 0.1 \mathrm{Myr}\right.$ at $\mathrm{z}=14)$. At later cosmic times, the average gas metallicity increases, so that the critical density for fragmentation decreases. A lower critical density implies that $R_{S F}$ increases (Equation 11), lengthening the core collapse timescale. Therefore, clusters form less concentrated and more massive and their core collapse timescale continues to increase with decreasing redshift. This behavior is evident in Fig. 4 for the mean quantities $\left(\left\langle M_{c l}\right\rangle,\left\langle R_{h}\right\rangle\right.$, $\left\langle t_{c c}\right\rangle$ and $\left.\left\langle M_{\mathrm{BH}}\right\rangle\right)$. The mean seed mass as a function of redshift is shown in Figure 4 (lower right panel). Unlike $\left\langle M_{c l}\right\rangle$ and $\left\langle R_{h}\right\rangle,\left\langle M_{\mathrm{BH}}\right\rangle$ shows no redshift dependence as the increase in $M_{c l}$ and $t_{c c}$ compensate (see Equation 15) leading to a roughly constant $M_{B H}$.

In Figure 5 the entire cluster mass function (solid histogram) is compared to the mass function of systems able to form a BH seed (dashed histogram). The clusters that do not form $\mathrm{BH}$ seeds are the very last to form, when the metallicity of the Universe is already significant. The BH mass function is also shown in Figure 5 , The mass function is peaked at $\simeq 1000 \mathrm{M}_{\odot}$ with a long tail at low masses, and a steep drop at high masses.

Fig. 8 shows the fraction, $f_{\mathrm{BH}}$, of halos hosting a $\mathrm{BH}$ seed. Seeds start to form at $z \sim 14$ in coincidence with the first, very compact, stellar clusters. The typical core collapse timescale increases with cosmic time, $\left\langle t_{c c}\right\rangle \sim 3$ Myr at $\mathrm{z} \sim 9$, and by $z \sim 8 f_{\mathrm{BH}}$ drops rapidly. From this point on even the most concentrated and least enriched disks are unable to create central stellar concentrations with $t_{c c}<3 \mathrm{Myr}$ and seed formation is completly suppressed. Figure 8 also shows the integrated comoving mass density of seeds $\rho_{\text {seed }}$. At $z \sim 10$ the mass density saturates at a value of $\sim 300 \mathrm{M}_{\odot} \mathrm{Mpc}^{-3}$. This mass density should be considered a lower limit for the total black holes mass density $\rho_{\mathrm{BH}}$ as we are completely neglecting $\mathrm{BH}$ growth after seeds formation. We will discuss the implications of this formation mechanism on the evolution of the supermassive black hole population in a forthcoming paper. We note however that this seed density is similar to that expected from PopIII star remnants (roughly a factor of 3 larger, Volonteri, Haardt \& Madau 2003), and we therefore expect that most observational constraints can be fulfilled at the same level.
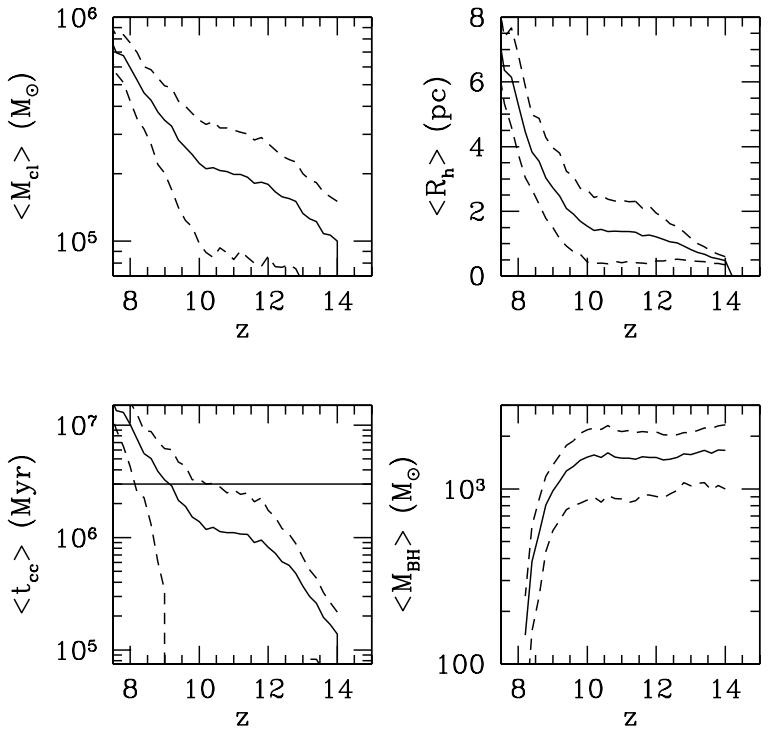

FIG. 4.- Mean cluster masses (upper left panel), radii (upper right), core collapse timescales (lower left) and $\mathrm{BH}$ masses (lower right) as a function of redshift for model $\mathrm{A}$. The orizontal line in the lower left panel marks the critical core collapse timescale for VMS formation. The dashed curves mark the $1-\sigma$ scatter.
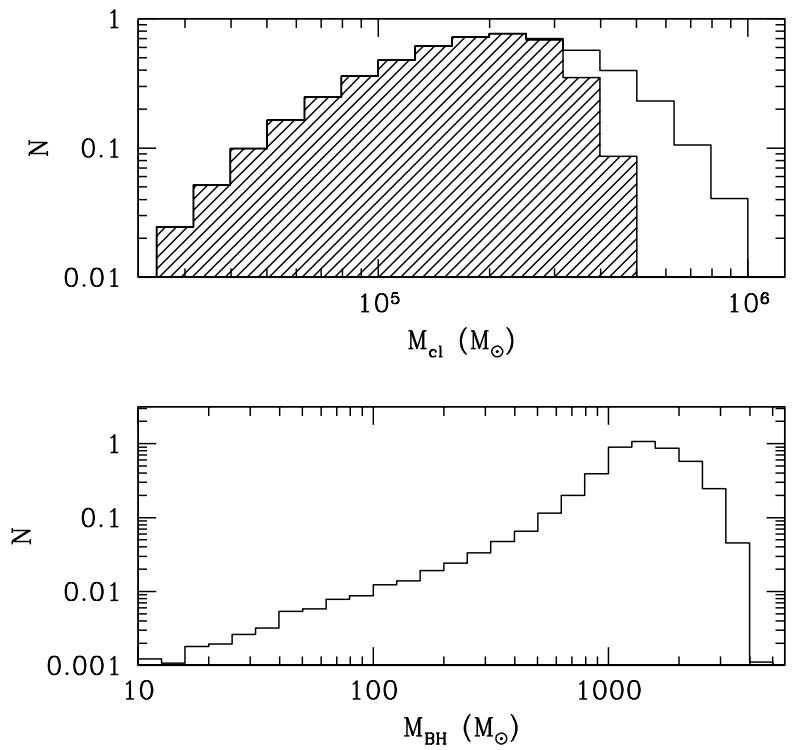

Fig. 5. - Top: Cluster mass function for model A integrated over all redshift. The shaded area corresponds to the subpopulation of clusters able to form $\mathrm{BH}$ seeds. Bottom: $\mathrm{BH}$ seed mass function integrated over all redshifts. 


\subsection{Impact of the uncertainties on metal enrichment onto the seed population}

We now discuss how our results depend on our choice of parameters. We compute the seed population for BHs formed over cosmic time varying (i) the history of metal enrichment (models $\mathrm{B}$ and $\mathrm{C}$ ) and (ii) the ratio of the coolants that determine the $Z_{c r i t}-n_{\text {crit }, Z}$ relationship (models D and E).

Mass functions of protogalactic nuclear clusters for all models defined in Table 1 are shown in Figure 2. Cluster stellar masses, $M_{c l}$, span 2 orders of magnitude, between $10^{4}-10^{6} \mathrm{M}_{\odot}$, with a peak around $\sim 10^{5} \mathrm{M}_{\odot}$. Figure 2 also shows the distribution of core collapse timescale of all clusters. The dashed vertical line at 3 Myr marks the limit for VMS formation. As discussed in the previous section, clusters satisfying the condition for the onset of runaway instability are clustered at small masses and radii: typical masses of runaway unstable clusters are around a few $10^{5} \mathrm{M}_{\odot}$ and typical radii are $\sim 1 \mathrm{pc}$ (see Equation [14). This naturally points towards the very first clusters: the first systems that form are indeed those that more easily can give birth to BH seeds. Consequently, these are also the most metal-poor clusters, so that our picture is consistent with requiring that VMS can more easily grow in low metallicity environments.

As times goes on, both $\left\langle M_{c l}\right\rangle$ and $\left\langle R_{h}\right\rangle$ grow: as a consequence $\left\langle t_{c c}\right\rangle$ increases. Even if clusters continue to form, BH seeds cannot be created any longer (cfr. Figure 7). The mass functions of $\mathrm{BH}$ seeds are shown in Figure 7 The seed mass distributions show a characteristic shape with a peak at a few $10^{3} M_{\odot}$ and a long tail at lower masses with very little redshift dependence. This general picture is valid for all models; we now discuss in turn the dependencies on specific model parameters.

\subsubsection{Changing $M E H$}

The MEH is one of the most uncertain parameters. We explore two extreme cases in models $\mathrm{B}(\beta=0.18$, blue dotted curves in Figure 6 and onwards) and $\mathrm{C}(\beta=0.36$ and $\Delta \log (Z)=0$, green dotted curves in Figure 6 and onwards). The MEH determines, together with the chosen $Z_{\text {crit }}-n_{\text {crit }, Z}$ relation, when seeds form. The duration of the $\mathrm{BH}$ seeds formation epoch is indeed given by a combination of the assumed metallicity spread, $\Delta \log (Z)$, and of the slope of the $Z(z)$. Once $Z$ increases over $Z_{c r i t, m i n}$, fragmentation is activated in a more extended region of the disk, the inflow is reduced and runaway instability can not proceed efficiently. The seed formation epoch is therefore longer either if $Z$ has larger $\Delta \log (Z)$ or if metal enrichment is rather inefficient.

Model B has the most efficient metal enrichment, and BHs appear already at redshift 30. On the other hand, $\mathrm{BH}$ formation is also suppressed very early, at $z \sim 18$. At this early cosmic epoch very few halos were massive enough for efficient atomic line cooling, thus leading to a comoving seed mass density of only a few $\mathrm{M}_{\odot}$. As previously noted for model A $\rho_{\text {seed }}$ does not necessary coincide with $\rho_{\mathrm{BH}}$ as we are neglecting seeds growth. In model $\mathrm{B}$ seeds form at higher redshift, and mass can be built up for a longer period, likely increasing $\mathrm{BH}$ masses by accretion.

Model $\mathrm{C}$ has the same redshift dependence of the $\mathrm{MEH}$ as our reference model A, but we assume no scatter. A
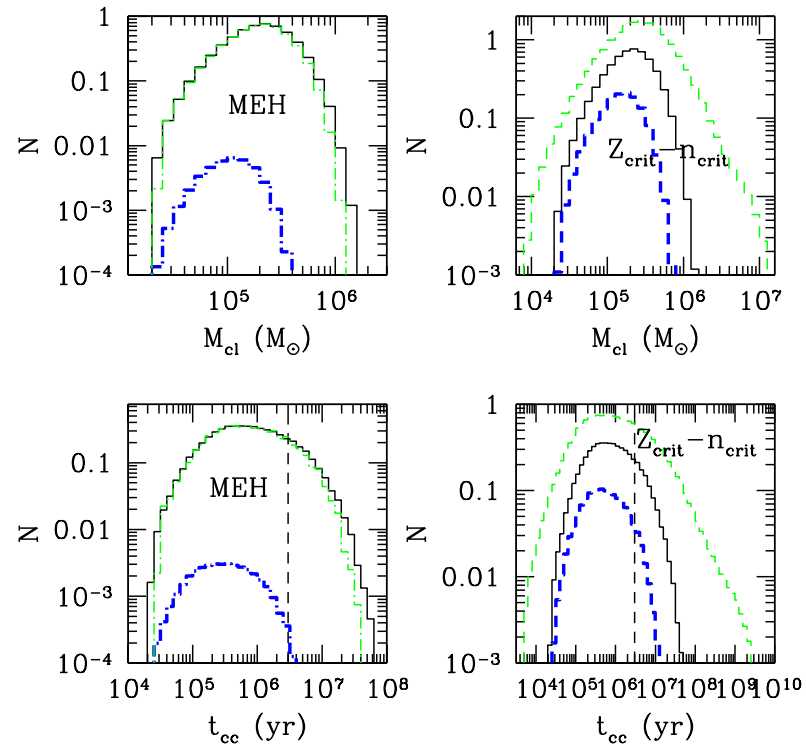

Fig. 6.- Top: Cluster mass functions integrated over all redshift. Bottom: distribution of core collapse timescales, integrated over all redshifts. Lines color and style as follow. Solid: black $=\mathrm{A}$. Dotted: thin $=\mathrm{B}$, thick $=\mathrm{C}$. Dashed: thick $=\mathrm{D}$, thin $=$ E. Model $\mathrm{A}$ is shown in all panels as a reference.

null $\Delta \log (Z)$ (model I) produces a short burst of seeds very concentrated in time, as seed formation is allowed only for a very sharp range of $Z$. Clusters and BHs form in a burst at $z=11-12$ when $Z \simeq 10^{-5}$ (the minimum in the $Z_{\text {crit }}-n_{\text {crit }, Z}$ relation). This burst is very efficient, with a high fraction of halos undergoing cluster and $\mathrm{BH}$ formation, and the resulting $\rho_{\text {seed }} \sim 300 \mathrm{M}_{\odot} \mathrm{Mpc}^{-3}$ is similar to our reference case.

\subsubsection{Changing $Z_{\text {crit }}-n_{\text {crit }, Z}$}

We explore the effect of different yield patterns for PopIII stars on our results in models D (blue long-dashed curves in Figure 6 and onwards) and E (green longdashed curves). The adopted $Z_{\text {crit }}-n_{\text {crit }, Z}$ relation defines together with the MEH, when seeds are born. The epoch at which $\mathrm{BH}$ seeds start to form is related to the redshift at which the first systems reach the minimum possible critical metallicity $Z_{\text {crit,min }} \simeq 10^{-5} Z_{\odot}$ (see the minimum of the curves in Fig. 10 of Santoro \& Shull 2007).

If fragmentation is allowed at lower metallicities (model D) clusters begin to form earlier. With increasing cosmic time, $R_{S F}$ increases to $R_{t r}$, thus precluding the formation of a seed. In model $\mathrm{D}$ this happens already at $z \sim 12$, while $\mathrm{BH}$ formation proceeds all the way to $z=5$ in model E. Consequently, the seed mass density $\rho_{\text {seed }}$ ranges from $\sim 100$ to $300 \mathrm{M}_{\odot} \mathrm{Mpc}^{-3}$ at $z=5$, with model $\mathrm{D}$, that forms seed early on, having the lower seed density.

\section{DISCUSSION}

We described a model for BH seed formation as result of multiple successive instabilities. On a large scale, grav- 

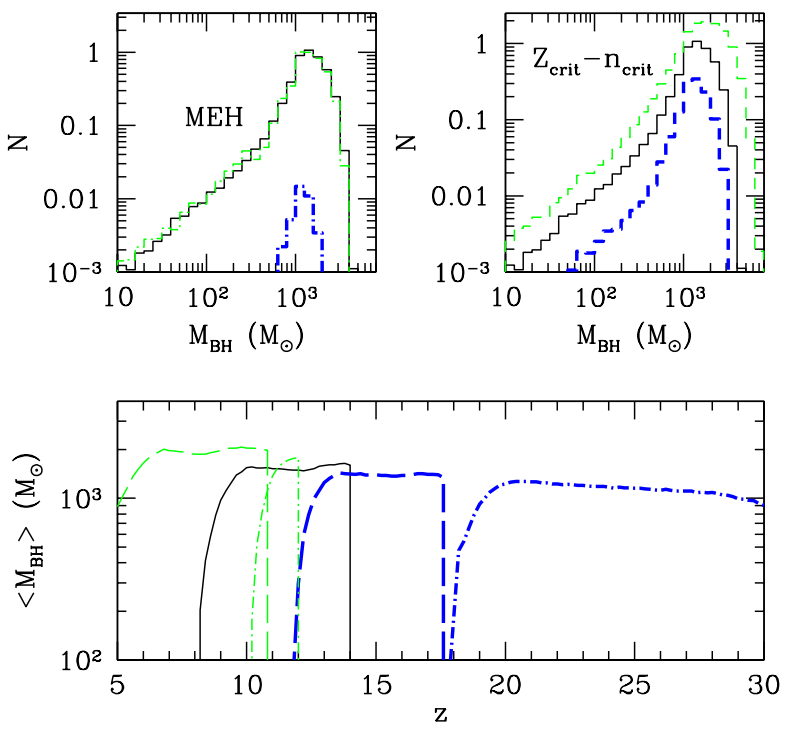

Fig. 7.- Top: Mass function of seed BHs, integrated over all redshifts. Bottom: mean $\mathrm{BH}$ mass as a function of redshift. Lines color and style as in Figure 6
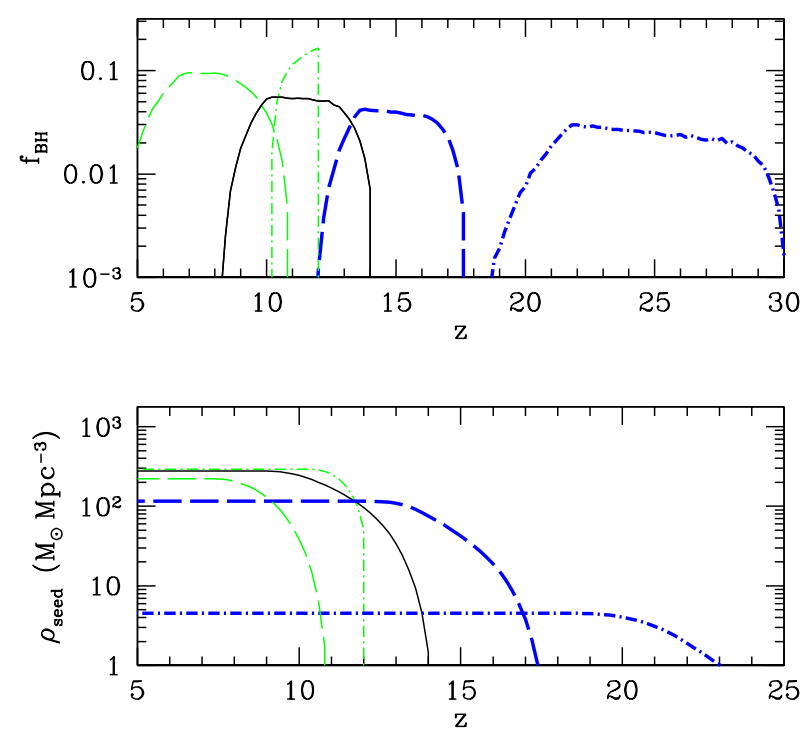

FIG. 8.- Top: fraction of halos hosting a BH seed as a function of redshift for models in Table 1. Bottom: Co-moving mass density of the seed $\mathrm{BH}$ for the same models. Colors and lines styles as in Figure 6 itational torques in Toomre unstable primordial disks can pile up a significant amount of gas in the central region of high redshift halos (e.g., Lodato \& Natarajan 2005). We focus on relatively massive halos at high redshift $\left(T_{\text {vir }}>10^{4} \mathrm{~K}, z \gtrsim 10\right)$ after the very first stars in the Universe have completed their evolution. This set of assumptions ensures that (i) atomic hydrogen cooling can contribute to the gas cooling process, (ii) a UV field from the first stars has heated the gas, precluding $\mathrm{H}_{2}$ cooling except in the highest density regions, and (iii) the gas inside the halo has been mildly polluted by the first metals. The second condition implies that, at least at the low density at which the collapse starts, $H_{2}$ is dissociated and does not contribute to the cooling of the gas (see also Figures 9 and 10 in O'Shea \& Norman 2008). The third condition ensures that metals (and dust) in small quantities can cool down the gas efficiently only as the gas density reaches a critical threshold. Jointly, the two conditions ensure that cooling is driven at first by atomic hydrogen only.

At low metallicity $\left(Z \sim 10^{-5} Z_{\odot}\right)$, fragmentation and low-mass star formation can occur only where the density is above a metallicity dependent critical threshold $\left(Z_{\text {crit }}-n_{\text {crit }, Z}\right.$, Santoro \& Shull 2006), corresponding to the central densest region of the protogalaxies. Within this limited region where star formation takes place efficiently, very compact stellar clusters form. The typical stellar masses are of order $10^{5} \mathrm{M}_{\odot}$ and the typical half mass radii $\sim 1$ pc. Eventually, a large fraction of these very dense clusters undergo core collapse before stars are able to complete stellar evolution and a VMS can form (e.g., Gürkan et al. 2004, Portegies Zwart et al. 2006).

Clusters unstable to runaway collisions are always the first, less massive $\left(\sim 10^{5} \mathrm{M}_{\odot}\right)$ ones. As the metallicity of the Universe increases, the critical density for fragmentation decreases and stars start to form in the entire protogalactic disk so that i) accretion of gas in the centre is no longer efficient and ii) the core collapse timescale increases. As a result less and less compact clusters form, and less of them are subject to rapid core collapse.

We computed the properties of the $\mathrm{BH}$ population for a set of models, in dependence of various parameters. The epoch of seed formation is determined by the time at which gas in the centre of the halo can start to fragment. This redshift depends on the metal enrichment history and on the exact shape of the $Z_{\text {crit }}-n_{\text {crit, } Z}$ relation. If metal pollution is very efficient and the Universe was enriched early, the $\mathrm{BH}$ formation epoch ends very early, when only a few halos were massive enough for efficient atomic line cooling. The mass and number densities of $\mathrm{BH}$ seeds are consequently very low. The fraction of halos hosting a $\mathrm{BH}$ seed depends also on the fraction of unstable disk (hence, the critical Toomre parameter, $Q_{c}$ ) and on the frequency of halos with $Z \sim Z_{\text {crit,min }}$. Decreasing $Q_{c}$ has a twofold effect on the efficiency of $\mathrm{BH}$ seeding. First, as already noted by Lodato \& Natarajan (2007), it decreases the number of bar-unstable disks as the Toomre criterion is satisfied for higher surface densities (requiring very small spin parameters). Additionally, the higher surface density for bar-instabilities implies smaller unstable regions $\left(R_{t r}\right)$, but a larger $R_{S F}$ at a fixed particle density. Cluster formation is therefore truncated at $\lambda<0.004$ for $Q_{c}=1$, leading to inefficient 
cluster and $\mathrm{BH}$ formation. The slope of the inner density profile, $\gamma$, affects $f_{\mathrm{BH}}$ as it affects the fraction of runaway unstable systems that can form a compact cluster. A lower $\gamma$ produces shallower, more extended clusters with longer core collapse timescales (Equations 11 and 14). For reasonable choices of $\gamma>4 / 3$ we find that the efficiency of the $\mathrm{BH}$ formation is within a factor of 2 of our reference model.

Most of our assumptions have been quite conservative, but still the population of seeds is comparable to the case of Population III star remnants discussed, for instance in Volonteri, Haardt \& Madau (2003). The fraction of highredshift galaxies seeded with a $\mathrm{BH}$ is about a factor of 10 below the direct collapse case presented in Volonteri, Lodato \& Natarajan (2008), where a seed was assumed to form with a $100 \%$ efficiency whenever a protogalaxy disk was Toomre unstable. An estimate of the degree of agreement between the evolution of the population of seeds that we have calculated in this paper and observational constraints requires dedicated models, including the mass growth of the BHs after their formation, and how galaxy and $\mathrm{BH}$ mergers influence the population. We will present such models in a future paper.

\section{ACKNOWLEDGEMENTS}

We are extremely grateful to Cole Miller for providing insightful comments on the manuscript. MV wishes to thank Marc Freitag for encouraging discussions during the very early stages of this work.

REFERENCES

Abel, T., Bryan, G. L., \& Norman, M. L. 2000, ApJ, 540, 39-44. Barkana, R., \& Loeb, A. 2001, Phys. Rep., 349, 125

Begelman, M. C., Volonteri, M., \& Rees, M. J. 2006, MNRAS, 370, 289

Begelman, M. C., \& Rees, M. J. 1978, MNRAS, 185, 847

Bett, P., Eke, V., Frenk, C. S., Jenkins, A., Helly, J., \& Navarro, J. 2007, MNRAS, 376, 215

Binney, J., \& Tremaine, S. 1987, Princeton, NJ, Princeton University Press, 1987, 747 p.,

Bromm, V., Ferrara, A., Coppi, P. S., \& Larson, R. B. 2001, MNRAS, 328, 969

Bromm, V., Coppi, P. S., \& Larson, R. B. 1999, ApJL, 527, L5-L8.

Bromm, V., Ferrara, A., Coppi, P. S., \& Larson, R. B. 2001, MNRAS, 328, 969

Bromm, V., Coppi, P. S., \& Larson, R. B. 2002, ApJ, 564, 23-51.

Bromm, V., \& Loeb, A. 2003, Nature, 425, 812

Ciardi, B. 2008, First Stars III, 990, 353

Ciardi, B., \& Ferrara, A. 2005, Space Science Reviews, 116, 625

Clark, P. C., Glover, S. C. O., \& Klessen, R. S. 2008, ApJ, 672, 757

Clarke, C. J., \& Bromm, V. 2003, MNRAS, 343, 1224

Clarke, C. J., Harper-Clark, E., \& Lodato, G. 2007, MNRAS, 381,1543

Di Matteo, T., Colberg, J., Springel, V., Hernquist, L., \& Sijacki, D. 2008, ApJ, 676, 33-53.

Dunkley, J., et al. 2008, ArXiv e-prints, 803, arXiv:0803.0586

Ebisuzaki, T., et al. 2001, ApJ, 562, L19

Eisenstein, D. J., \& Loeb, A. 1995, ApJ, 443, 11

Fan, X., et al. 2001, AJ, 122, 2833

Freese, K., Bodenheimer, P., Spolyar, D., \& Gondolo, P. 2008, ArXiv e-prints, 806, arXiv:0806.0617

Freitag, M., Gürkan, M. A., \& Rasio, F. A. 2006, MNRAS, 368, 141

Freitag, M., Rasio, F. A., \& Baumgardt, H. 2006, MNRAS, 368, 121

Gammie, C. F. 1996, ApJ, 462, 725

Gürkan, M. A., Freitag, M., \& Rasio, F. A. 2004, ApJ, 604, 632

Haehnelt, M. G. \& Rees, M. J., MNRAS 263, 168-178.

Haiman, Z. \& Menou, K. 2000, ApJ, 531, 42-51.

Heger, A., Fryer, C. L., Woosley, S. E., Langer, N., \& Hartmann,

D. H. 2003, ApJ, 591, 288

Hirschi, R. 2007, A\&A, 461, 571

Kauffmann, G. \& Haehnelt, M. 2000, MNRAS, 311, 576-588.

Koushiappas, S. M., Bullock, J. S., \& Dekel, A. 2004, MNRAS, 354,292

Kulkarni, V. P., Fall, S. M., Lauroesch, J. T., York, D. G., Welty, D. E., Khare, P., \& Truran, J. W. 2005, Apj, 618, 68

Lada, C. J., \& Lada, E. A. 2003, ARA\&A, 41, 57

Li, L.-X. 2007, ArXiv e-prints, 710, arXiv:0710.3587

Lodato, G., \& Natarajan, P. 2006, MNRAS, 371, 1813

Lodato, G., \& Natarajan, P. 2007, MNRAS, 377, L64

Loeb, A., \& Rasio, F. A. 1994, ApJ, 432, 52
Madau, P., \& Rees, M. J. 2001, ApJ, 551, L27

Marchant, A. B., \& Shapiro, S. L. 1980, ApJ, 239, 685

Meynet, G., et al. 2008, IAU Symposium, 250, 571

Miller, M. C., \& Hamilton, D. P. 2002, MNRAS, 330, 232

Mineshige, S., \& Umemura, M. 1997, ApJ, 480, 167

Mo, H. J., Mao, S., \& White, S. D. M. 1998, MNRAS, 295, 319

Natarajan, A., Tan, J. C., \& O'Shea, B. W. 2008, ArXiv e-prints, 807, arXiv:0807.3769

Oh, S. P., \& Haiman, Z. 2002, ApJ, 569, 558

Omukai, K., Schneider, R., \& Haiman, Z. 2008, ArXiv e-prints, 804, arXiv:0804.3141

Omukai, K., Tsuribe, T., Schneider, R., \& Ferrara, A. 2005, ApJ, 626,627

O'Shea, B. W., \& Norman, M. L. 2008, ApJ, 673, 14

Portegies Zwart, S. F., Makino, J., McMillan, S. L. W., \& Hut, P. 1999, A\&A, 348, 117

Portegies Zwart, S. F., Baumgardt, H., Hut, P., Makino, J., \&

McMillan, S. L. W. 2004, Nature, 428, 724

Portegies Zwart, S. F., \& McMillan, S. L. W. 2002, ApJ, 576, 899

Prochaska, J. X., Chen, H.-W., Dessauges-Zavadsky, M., \& Bloom, J. S. 2007, Apj, 666, 267

Prochaska, J. X., Gawiser, E., Wolfe, A. M., Castro, S., \& Djorgovski, S. G. 2003, Apj, 595, L

Rice, W. K. M., Lodato, G., \& Armitage, P. J. 2005, MNRAS, 364, L56

Santoro, F., \& Shull, J. M. 2006, ApJ, 643, 26

Savaglio, S. 2006, New Journal of Physics, 8, 195

Savaglio, S., et al. 2005, Apj, 635, 260

Scannapieco, E., Schneider, R., \& Ferrara, A. 2003, ApJ, 589, 35

Scannapieco, E., Ferrara, A., \& Madau, P. 2002, ApJ, 574, 590

Schneider, R., Ferrara, A., Salvaterra, R., \& Omukai, K. 2004, Bulletin of the American Astronomical Society, 36, 704

Schneider, R., Omukai, K., Inoue, A. K., \& Ferrara, A. 2006, MNRAS, 369, 1437

Shakura, N. I., \& Sunyaev, R. A. 1973, A\&A, 24, 337

Sheth, R. K., \& Tormen, G. 1999, MNRAS, 308, 119

Shlosman, I., Begelman, M. C., \& Frank, J. 1990, Nature, 345, 679

Smith, B. D., Turk, M. J., Sigurdsson, S., O'Shea, B. W., \& Norman, M. L. 2008, ArXiv e-prints, 806, arXiv:0806.1653

Spolyar, D., Freese, K., \& Gondolo, P. 2008, Physical Review Letters, 100, 051101

Tornatore, L., Ferrara, A., \& Schneider, R. 2007, MNRAS, 382, 945

Tumlinson, J., Venkatesan, A., \& Shull, J. M. 2004, ApJ, 612, 602

Volonteri, M., Haardt, F., \& Madau, P. 2003, ApJ, 582, 559

Volonteri, M., \& Rees, M. J. 2006, ApJ, 650, 669

Volonteri, M., Lodato, G., \& Natarajan, P. 2008, MNRAS, 383, 1079

Wyithe, J. S. B. \& Loeb, A. 2003, ApJ, 595, 614-623. 


\section{APPENDIX}

\section{DISK AND CLUSTER STRUCTURE}

\section{Disk parameters}

Disk parameters $\Sigma_{0}, R_{0}, R_{t r}$ and $m_{a}$ have been summarized in Section 2.1. We only insert here the complete expression for $R_{t r}$ with the $\gamma$ dependency

$$
R_{t r}=\frac{2-\gamma}{\gamma-1} \frac{m_{a} M_{h}}{2 \pi \Sigma_{0} R_{0}}
$$

As stated in Section 2.1, the particle density of the inner disk can be written as

$$
n(R, h)=n_{0} \frac{R_{0}}{R_{t r}}\left(\frac{R_{t r}}{R}\right)^{\gamma} \cosh ^{-2}(h / H(R))
$$

where $H$ can be computed imposing hydrostatic equilibrium and it is found out to be $H=\frac{c_{s}}{\sqrt{2 \pi G \mu m_{u} n_{0} f(R)}}$ (see for example Oh \& Haiman 2002). To simplify the notation we have defined $f(R)=R_{0} / R_{t r}\left(R_{t r} / R\right)^{\gamma}$. We relate $n_{0}$ and $\Sigma_{0}$ by imposing that the surface density calculated starting from Equation A2, follows the profile described by Equation 3:

$$
\begin{aligned}
\Sigma_{0} f(R) & =\mu m_{u} n_{0} f(R) \int_{-\infty}^{+\infty} \cosh ^{-2}(z / h) \mathrm{d} z \\
& =2 \mu m_{u} n_{0} f(R) H=\sqrt{2} c_{s} \sqrt{\frac{n_{0} \mu m_{u} f(R)}{\pi G}} .
\end{aligned}
$$

Resolving for $n_{0}$ we find:

$$
n_{0}=\frac{\Sigma_{0}^{2}}{c_{s}^{2}} \frac{\pi G f(R)}{2 \mu m_{u}} .
$$

Star formation radius

Once the density profile of the disk is defined, the star formation radius can be determined by imposing $n(R=$ $\left.R_{S F}, h=0\right)=n_{\text {crit }, Z}$. We allow clusters to form only if star formation is induced inside $R_{t r}$ and not in the external part of the disk. The condition for this to happen is that $n_{t r} \equiv n\left(R=R_{t r}, h=0\right)<n_{c r i t, Z}$. $R_{S F}$ is then computed by imposing:

$$
n_{0} \frac{R_{0}}{R_{t r}}\left(\frac{R_{t r}}{R_{S F}}\right)^{\gamma}=n_{c r i t, Z}
$$

Inserting the expression for $n_{0}$

$$
\frac{\pi G \Sigma_{0}^{2}}{2 \mu m_{u} c_{s}^{2}}\left(\frac{R_{0}}{R_{t r}}\right)^{2}\left(\frac{R_{t r}}{R_{S F}}\right)^{2 \gamma}=n_{c r i t, Z}
$$

Resolving for $R_{S F}$

$$
\begin{aligned}
R_{S F} & =R_{t r}\left[\frac{\Sigma_{0} R_{0}}{c_{s} R_{t r}} \sqrt{\frac{\pi G}{2 \mu m_{u} n_{c r i t, Z}}}\right]^{1 / \gamma} \\
& =R_{t r}^{2 / 5}\left[\frac{\Sigma_{0} R_{0}}{c_{s}} \sqrt{\frac{\pi G}{2 \mu m_{u} n_{c r i t, Z}}}\right]^{3 / 5}
\end{aligned}
$$

where in last expression we have inserted $\gamma=5 / 3$ explicitly.

As stated in Section 2.3, $n_{\text {crit }, Z}$ depends on the metallicity of the gas. The curves in Figure 10 of Santoro \& Shull can be fitted by the expression

$$
\log \left(Z / Z_{\odot}\right)=a \log ^{2}\left(n_{c r i t, Z}\right)+b \log \left(n_{c r i t, Z}\right)+c
$$

where the values of $a, b$, and $c$ have been calculated for the three curves and are reported in Table 2

The minimal critical density for fragmentation can be computed from Equation A8. Inserting the expression for the critical density into Equation A7 one finds 


\begin{tabular}{|c|c|c|c|}
\hline$Z_{\text {crit }}-n_{\text {crit }}$ & 1 & 2 & 3 \\
\hline \hline $\mathrm{a}$ & 0.03517305 & 0.0317305 & 0.0317305 \\
\hline $\mathrm{b}$ & -0.582132 & -0.572132 & -0.62132 \\
\hline $\mathrm{c}$ & -4.1 & -2.75 & -1.1 \\
\hline
\end{tabular}

TABLE 2

List of the VAlues of $a, b$ AND $c$ FOR FitTing Different $Z_{\text {crit }}-n_{\text {crit }}$ CURVES.

$$
\begin{aligned}
\log \left(R_{S F}\right)= & \frac{\gamma-1}{\gamma} \log \left(\frac{2-\gamma}{\gamma-1} \frac{m_{a} M_{h}}{2 \pi \Sigma_{0} R_{0}}\right)+ \\
& \frac{1}{\gamma}\left[\log \left(\frac{\Sigma_{o} R_{o}}{c_{s}}\right)+\frac{1}{2} \log \left(\frac{\pi G}{2 \mu m_{u}}\right)-\frac{1}{2} \log \left(n_{c r i t, Z}\right)\right] \\
= & \frac{\gamma-1}{\gamma}\left[\log \left(\frac{2-\gamma}{\gamma-1}\right)+A\right]+ \\
& \frac{1}{\gamma}\left(B-C^{\prime} \sqrt{1+D^{\prime} \log \left(Z / Z_{\odot}\right)}\right) \\
= & \frac{\gamma-1}{\gamma}\left[\log \left(\frac{2-\gamma}{\gamma-1}\right)+A\right]+ \\
& \frac{1}{\gamma}(B-C \sqrt{1+D \beta z})
\end{aligned}
$$

where in the last expression we have first expressed $n_{c r i t, Z}$ as a function of $Z$ and then we have inserted the redshift dependence of the metallicity ${ }^{1} Z$. All constants except $\gamma$ have been included in $A, B, C, C^{\prime}, D$ and $D^{\prime}$ :

$$
\begin{aligned}
A & =\log \left(\frac{m_{a} M_{h}}{2 \pi \Sigma_{0} R_{0}}\right) \\
B & =\frac{b}{4 a}+\log \left(\frac{\Sigma_{0} R_{0}}{c_{s}}\right)+\frac{1}{2} \log \left(\frac{\pi G}{2 \mu m_{u}}\right) \\
C^{\prime} & =\frac{b}{4 a} \sqrt{1-\frac{4 a c}{b^{2}}} \\
C & =C^{\prime} \sqrt{1+D^{\prime} \delta} \\
D^{\prime} & =\frac{4 a}{b^{2}-4 a c} \\
D & =-\frac{D^{\prime}}{1+D^{\prime} \delta}
\end{aligned}
$$

Finally for $\gamma=5 / 3$

$$
\log \left(R_{S F}\right)=k_{1}+k_{2} \sqrt{1+k_{3} \beta z}
$$

where all constants are collected in $k_{1} \equiv \frac{2}{5}(A-0.3)+3 / 5 B, k_{2} \equiv 3 / 5 C$ and $k_{3} \equiv D$.

Black hole masses

Black hole masses depend on the stellar mass of the clusters and on the half-mass radius, via the core collapse timescale.

Cluster masses and timescales to core collapse can be estimated coupling Equations A7 and A11 to Equation 12:

$$
\begin{aligned}
M_{c l} & =2 \pi \epsilon \int_{0}^{R_{S F}} \Sigma_{0} R_{0} R_{t r}^{\gamma-1} R^{1-\gamma} \mathrm{d} R \\
& =2 \pi \epsilon_{S F} \Sigma_{0} R_{0} R_{t r}^{\gamma-1} \frac{R_{S F}^{2-\gamma}}{2-\gamma}
\end{aligned}
$$

1 The last expression refers to the mean $Z$ only, i.e. it is rigorously appropriate only to model I 


$$
\begin{aligned}
& =2 \pi \epsilon_{S F} \Sigma_{0} R_{0}\left[\frac{2-\gamma}{\gamma-1} \frac{m_{a} M_{h}}{2 \pi \Sigma_{0} R_{0}}\right]^{\gamma-1} \frac{R_{S F}^{2-\gamma}}{2-\gamma} \\
& =\epsilon_{S F}\left[\frac{2 \pi}{2-\gamma} \frac{\Sigma_{0}^{2} R_{0}^{2}}{c_{s}} \sqrt{\frac{\pi G}{2 \mu m_{u} n_{c r i t}}}\right]^{\frac{2-\gamma}{\gamma}}\left(\frac{m_{a} M_{h}}{\gamma-1}\right)^{\frac{2 \gamma-2}{\gamma}} \\
& =\epsilon_{S F}\left[6 \pi \frac{\Sigma_{0}^{2} R_{0}^{2}}{c_{s}} \sqrt{\frac{\pi G}{2 \mu m_{u} n_{\text {crit }}}}\right]^{1 / 5}\left(\frac{3}{2} m_{a} M_{h}\right)^{4 / 5}
\end{aligned}
$$

where in the last line we assume $\gamma=5 / 3$.

The core collapse timescale is defined in Equation [14. The half mass radius can be simply expressed as $R_{h}=$ $2^{1 /(\gamma-1)} R_{S F}$. We use Equation A11 to express the dependence of $t_{c c}$ on $M_{c l}$ as:

$$
\begin{aligned}
t_{c c} & =\tau_{0} R_{h}^{3 / 2} M_{c l}^{1 / 2}=\tau_{0} 2^{\frac{3}{2} \frac{1}{\gamma-1}} R_{S F}^{3 / 2} M_{c l}^{1 / 2}=\tau_{0} 2^{\frac{3}{2} \frac{1}{\gamma-1}} \\
& \times\left[\frac{2-\gamma}{2 \pi \Sigma_{0} R_{0} \epsilon_{S F}^{1 /(2-\gamma)}}\right]^{3 / 2}\left(\frac{m_{a} M_{h}}{\gamma-1}\right)^{\frac{3}{2} \frac{1-\gamma}{2-\gamma}} M_{c l}^{\frac{5-\gamma}{2(2-\gamma)}} \\
& =\tau_{0} 2^{9 / 4}\left[\frac{1}{6 \pi \Sigma_{0} R_{0} \epsilon_{S F}^{3}}\right]^{3 / 2}\left(\frac{3}{2} m_{a} M_{h}\right)^{-3} M_{c l}^{5}
\end{aligned}
$$

where $\tau_{0}$ is the normalization of Equation[14 and its value in $\mathrm{Myr}, \mathrm{M}_{\odot}$ and $\mathrm{pc}$ is 0.019 . In the last Equation we assume $\gamma=5 / 3$.

$M_{\mathrm{BH}}$ can now be found by inserting Equation A14 into Equation 15

$$
\begin{aligned}
M_{\mathrm{BH}} & =m_{*}+4 \cdot 10^{-3} f_{c} \ln \lambda M_{c l} \ln \left(\frac{g}{M_{c l}^{\frac{5-\gamma}{2(2-\gamma)}}}\right) \\
& \propto M_{c l} \ln \frac{\tau}{M_{c l}^{5}}
\end{aligned}
$$

where $g$ is defined as

$$
g=\frac{t_{\mathrm{MS}}}{\tau_{0}} 2^{-\frac{3}{2} \frac{1}{\gamma-1}}\left[\frac{2-\gamma}{2 \pi \epsilon_{S F}^{2-\gamma} \Sigma_{0} R_{0}}\right]^{-3 / 2}\left(\frac{m_{a} M_{h}}{\gamma-1}\right)^{-\frac{3}{2} \frac{1-\gamma}{2-\gamma}} .
$$

From Equation $\mathrm{A15}$ we can argue that $M_{\mathrm{BH}}$ first grows with increasing $M_{c l}$, it attains a maximum value and then it decreases, as more massive clusters have longer core collapse timescales with $t_{c c} \propto M_{c l}^{5}$. This last proportionality results from the dependence of $R_{S F}$ (and consequently $R_{h}$ ) on $M_{c l}$. 